\title{
Fibrations in the Category of Absolute Neighborhood Retracts
}

by

Takahisa MIYATA

Presented by Czestaw BESSAGA

\begin{abstract}
Summary. The category Top of topological spaces and continuous maps has the structures of a fibration category and a cofibration category in the sense of Baues, where fibration $=$ Hurewicz fibration, cofibration $=$ the usual cofibration, and weak equivalence $=$ homotopy equivalence. Concentrating on fibrations, we consider the problem: given a full subcategory $\mathcal{C}$ of Top, is the fibration structure of Top restricted to $\mathcal{C}$ a fibration category? In this paper we take the special case where $\mathcal{C}$ is the full subcategory ANR of Top whose objects are absolute neighborhood retracts. The main result is that ANR has the structure of a fibration category if fibration = map having a property that is slightly stronger than the usual homotopy lifting property, and weak equivalence $=$ homotopy equivalence.
\end{abstract}

1. Introduction. The best known approach to axiomatic homotopy theory is Quillen's model category [3]. Baues [1] introduced the notions of a fibration category and a cofibration category. Those notions make the constructions of homotopy theory available in more contexts by simply weakening the assumptions and concentrating on either fibrations or cofibrations.

The category Top of topological spaces and continuous maps is the best known example of a fibration category and a cofibration category. Typical structures of the fibration category and the cofibration category consist of Hurewicz fibrations, the usual cofibrations, and homotopy equivalences (see [1, Theorem 5.1, p. 34; Theorem 5.2, p. 35], for example).

Concentrating on the structure of a fibration category for Top, we consider the following problem: given a full subcategory $\mathcal{C}$ of Top, is the fibration structure of Top restricted to $\mathcal{C}$ a fibration category?

2000 Mathematics Subject Classification: 54C55, 55U35, 55P30.

Key words and phrases: ANR, fibration category, strong homotopy lifting property. 
We observe that if $\mathcal{C}$ is a full subcategory of Top which has the following properties: (i) $X, Y \in \mathcal{C} \Rightarrow X \times Y \in \mathcal{C}$; (ii) $X \in \mathcal{C} \Rightarrow X^{I} \in \mathcal{C}$; (iii) $X \in \mathcal{C}$, and $A \subseteq X$ is closed $\Rightarrow A \in \mathcal{C}$, then the fibration structure of Top restricted to $\mathcal{C}$ is a fibration category. Here, $I=[0,1]$. For example, the full subcategory $M$ of Top whose objects are metric spaces satisfies those properties.

In this paper we take the special case where $\mathcal{C}$ is the full subcategory ANR of Top whose objects are absolute neighborhood retracts (ANR's). ANR's are useful in many areas of topology. For example, shape theory is in some sense an extension of the usual homotopy theory restricted to ANR [2]. Thus it is a natural question whether the restriction to ANR has the structure of a fibration category.

We observe that ANR fails to have property (iii), so it is not automatic that the restriction of the fibration structure on Top to ANR is a fibration category. In this paper we show that it becomes a fibration category if we take for fibrations maps having a property that is slightly stronger than the usual homotopy lifting property.

A map $p: E \rightarrow B$ is said to have the strong homotopy lifting property (SHLP) with respect to a space $X$ provided if $h: X \rightarrow E$ and $H: X \times I \rightarrow B$ are maps such that

$$
p h=H_{0},
$$

there is a map $\tilde{H}: X \times I \rightarrow E$ such that

$$
h=\tilde{H}_{0}, \quad p \tilde{H}=H,
$$

and whenever $H$ is constant on $x \times I, \tilde{H}$ is constant on $x \times I$.

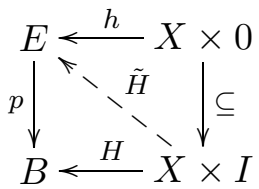

If a Hurewicz fibration has the unique path lifting property, it has the SHLP with respect to every space (see [4, Theorem 5, p. 68]).

The following are the main results of the paper:

THEOREM A. ANR is a fibration category if fibration = map having the SHLP with respect to every space, and weak equivalence $=$ homotopy equivalence.

TheOREM B. The full subcategory $\mathrm{M}$ of Top whose objects are metric spaces is a fibration category if fibration = map having the SHLP with respect to every space, and weak equivalence $=$ homotopy equivalence.

A fibration category is a category $\mathcal{F}$ with the structure $(\mathcal{F}, f i b$, we) which satisfies axioms (F1)-(F4) below. Here fib and we are classes of morphisms, 
called fibrations and weak equivalences, respectively. For more details, the reader is referred to [1].

(F1) Composition axiom. The isomorphisms in $\mathcal{F}$ are weak equivalences and fibrations. For any morphisms $f: X \rightarrow Y$ and $g: Y \rightarrow Z$, if any two of $f, g$, and $g f$ are weak equivalences, so is the third. The composition of fibrations is a fibration.

(F2) Pull-back axiom. For any 2-sink $B \stackrel{g}{\rightarrow} Y \stackrel{f}{\leftarrow} X$ in $\mathcal{F}$ with $f$ being a fibration, there is a pull-back diagram in $\mathcal{F}$

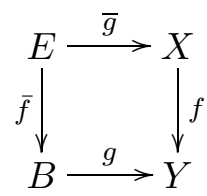

where $\bar{f}$ is a fibration. Moreover, if $f$ (resp., $g$ ) is a weak equivalence, so is $\bar{f}$ (resp., $\bar{g}$ ).

(F3) Factorization axiom. Each morphism $f: X \rightarrow Y$ admits a factorization

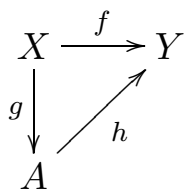

where $g$ is a weak equivalence and $h$ is a fibration.

(F4) Axiom on cofibrant models. Each object $X$ in $\mathcal{F}$ admits a trivial fibration (i.e., a morphism which is both a fibration and a weak equivalence) $R X \rightarrow X$ where $R X$ is a cofibrant in $\mathcal{F}$. An object $R$ is a cofibrant if each trivial fibration $f: Q \rightarrow R$ admits a morphism $s: R \rightarrow Q$ such that $f s=1_{R}$.

A cofibration category is a category $\mathcal{F}$ with the structure $(\mathcal{F}$, cof, we), where cof and we are classes of morphisms in $\mathcal{F}$, called cofibrations and weak equivalences, respectively, and it satisfies the condition that the opposite category $\mathcal{C}=\mathcal{F}^{\mathrm{op}}$ is a fibration category, where the structure of $\mathcal{C}$ is given by

- $f^{\text {op }}$ is a fibration in $\mathcal{C}$ iff $f$ is a cofibration in $\mathcal{F}$,

- $f^{\text {op }}$ is a weak equivalence in $\mathcal{C}$ iff $f$ is a weak equivalence in $\mathcal{F}$.

Throughout the paper, space means topological space, and map means continuous map.

For any space $X$ with a metric $\mathrm{d}$, for any $\varepsilon>0$, and for any $x \in X$, let $B_{\varepsilon}(x)=\{y \in X: \mathrm{d}(x, y)<\varepsilon\}$. For any open covering $\mathcal{V}$ of a space $Y$, two points $y, y^{\prime}$ of $Y$ are $\mathcal{V}$-near, denoted $\left(y, y^{\prime}\right)<\mathcal{V}$, provided $y, y^{\prime} \in V$ for some $V \in \mathcal{V}$, and two maps $f, g: X \rightarrow Y$ are $\mathcal{V}$-near, denoted $(f, g)<\mathcal{V}$, provided $f(x)$ and $g(x)$ are $\mathcal{V}$-near for each $x \in X$. For any open covering $\mathcal{U}$ of a space 
$X$ and for any subset $A$ of $X$, let $\operatorname{st}(A, \mathcal{U})=\bigcup\{U \in \mathcal{U}: U \cap A \neq \emptyset\}$, and let st $\mathcal{U}$ be the open covering $\{\operatorname{st}(U, \mathcal{U}): U \in \mathcal{U}\}$ of $X$. For any coverings $\mathcal{U}$ and $\mathcal{V}$ of a space $X, \mathcal{U}$ is a refinement of $\mathcal{V}$, denoted $\mathcal{U}<\mathcal{V}$, provided for each $U \in \mathcal{U}$ there exists $V \in \mathcal{V}$ such that $U \subseteq V$.

Let $X$ be a space with a metric d. For any path $\alpha: I \rightarrow X$, the diameter $|\alpha|$ of $\alpha$ is defined as the diameter of the image of $\alpha$, i.e.,

$$
|\alpha|=\sup \left\{\mathrm{d}\left(\alpha(t), \alpha\left(t^{\prime}\right)\right): t, t^{\prime} \in I\right\} .
$$

Then the function $X^{I} \rightarrow \mathbb{R}_{\geq 0}: \alpha \mapsto|\alpha|$ is continuous, and $|\alpha|=0$ iff $\alpha$ is a constant path.

\section{Proof of Theorem A}

TheOREM 2.1. Every map $f: A \rightarrow B$ between ANR's is the composition of a homotopy equivalence $q: A \rightarrow E$ and a map $p: E \rightarrow B$ having the $S H L P$ with respect to every space, where $E$ is an ANR.

Proof. Let $B$ be embedded in a convex subset $K$ of a normed linear space. Then there exist an open neighborhood $N$ of $B$ in $K$ and a retraction $r: N \rightarrow B$. Let $\mathcal{W}$ be the set of open balls $W$ in $K$ such that $W \subseteq N$, and let $\mathcal{V}=\{W \cap B: W \in \mathcal{W}\}$. Set $E=\left\{(x, \omega) \in A \times B^{I}:(f(x), \omega(0))<\mathcal{V}\right\}$. Note that $E$ is an ANR since it is an open subset of the ANR $A \times B^{I}$. Define a map $\varrho: E \rightarrow B^{I}$ by

$$
\varrho(x, \omega)(t)=r((1-t) f(x)+t \omega(0)) .
$$

Then for each $(x, \omega) \in E, \varrho(x, \omega)$ is a path from $f(x)$ to $\omega(0)$ in $B$. The map $f$ factors into the composition of $q: A \rightarrow E$ and $p: E \rightarrow B$ defined by $q(x)=\left(x, e_{f(x)}\right)$ for $x \in X$ and $p(x, \omega)=\omega(1)$ for $(x, \omega) \in E$, respectively. Here for any $y_{0} \in B, e_{y_{0}}$ denotes the constant map $I \rightarrow B: e_{y_{0}}(t)=y_{0}$.

Now, $q$ is a homotopy equivalence. Indeed, if $p_{A}: E \rightarrow A$ is the restriction of the projection map of $A \times B^{I}$ onto $A$, then $p_{A} q=1_{A}$, and $q p_{A} \simeq 1_{E}$, with the homotopy $H: E \times I \rightarrow E$,

$$
H((x, \omega), t)= \begin{cases}\left(x, e_{\varrho(x, \omega)(2 t)}\right), & 0 \leq t \leq 1 / 2, \\ \left(x, \omega_{2 t-1}\right), & 1 / 2 \leq t \leq 1 .\end{cases}
$$

Here, for each $t \in I$, the path $\omega_{t}: I \rightarrow B$ is defined by $\omega_{t}(s)=\omega(s t)$ for $s \in I$.

It remains to show that $p$ has the SHLP with respect to every space $X$. Suppose that we are given a commutative diagram

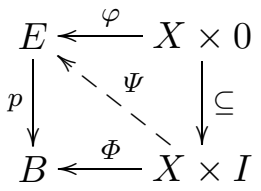


Write $\varphi(x)=\left(\varphi_{1}(x), \varphi_{2}(x)\right) \in A \times B^{I}$. Then the diagram can be completed by the map $\Psi: X \times I \rightarrow E, \Psi(x, t)=\left(\Psi_{1}(x, t), \Psi_{2}(x, t)\right) \in A \times B^{I}$, defined as follows. Let $\Psi_{1}(x, t)=\varphi_{1}(x)$. For each $(x, t) \in X \times I$ with $\left|\varphi_{2}(x)\right| \neq 0$ or $\left|\Phi_{t}(x)\right| \neq 0$, set

$$
\mu(x, t)=\frac{\left|\varphi_{2}(x)\right|}{\left|\varphi_{2}(x)\right|+\left|\Phi_{t}(x)\right|}, \quad \nu(x, t)=\frac{\left|\Phi_{t}(x)\right|}{\left|\varphi_{2}(x)\right|+\left|\Phi_{t}(x)\right|} .
$$

Here, for each $(x, t) \in X \times I, \Phi_{t}(x) \in B^{I}$ is defined by

$$
\Phi_{t}(x)(u)=\Phi(x, t u) \quad \text { for } u \in I .
$$

Then for each $(x, t) \in X \times I$, define the path $\Psi_{2}(x, t)$ by setting, for each $u \in I$,

$$
\Psi_{2}(x, t)(u)=\left\{\begin{array}{c}
\varphi_{2}(x)\left(\frac{u}{\mu(x, t)}\right) \\
(0 \leq u \leq \mu(x, t)) \\
\Phi_{t}(x)\left(\frac{u-\mu(x, t)}{\nu(x, t)}\right) \\
(\mu(x, t)<u \leq 1)
\end{array}\right\} \text { if }\left|\varphi_{2}(x)\right| \neq 0 \text { and }\left|\Phi_{t}(x)\right| \neq 0,
$$

If $\Phi$ is constant on $x \times I$, then $\left|\Phi_{t}(x)\right|=0$ for $t \in I$, so by the definition, $\Psi$ is constant on $x \times I$. It remains to show that $\Psi$ is continuous. Fix $(x, t) \in X \times I$, and let $\varepsilon>0$. Take $\xi>0$ with $0<\xi<\min \{1, \varepsilon\}$ such that if $\left|u-u^{\prime}\right|<\xi$ $\left(u, u^{\prime} \in I\right)$, then

$$
\begin{aligned}
& \mathrm{d}\left(\varphi_{2}(x)(u), \varphi_{2}(x)\left(u^{\prime}\right)\right)<\varepsilon / 3, \\
& \mathrm{~d}\left(\Phi_{t}(x)(u), \Phi_{t}(x)\left(u^{\prime}\right)\right)<\varepsilon / 3 .
\end{aligned}
$$

Also take $\delta_{1}>0$ such that if $\mathrm{d}\left(x, x^{\prime}\right)<\delta_{1}\left(x^{\prime} \in X\right)$ and $\left|t-t^{\prime}\right|<\delta_{1}\left(t^{\prime} \in I\right)$, then

$$
\begin{aligned}
& \mathrm{d}\left(\varphi_{2}(x), \varphi_{2}\left(x^{\prime}\right)\right)<\varepsilon / 3, \\
& \mathrm{~d}\left(\Phi_{t}(x), \Phi_{t^{\prime}}\left(x^{\prime}\right)\right)<\varepsilon / 3 .
\end{aligned}
$$

Choose $\xi^{\prime}>0$ with the following properties:

$$
\begin{aligned}
& \xi^{\prime}<\xi / 2 \\
& \xi^{\prime}<\frac{1}{8} \min \left\{\frac{1}{\mu(x, t)} \xi, \frac{\nu(x, t)}{\mu(x, t)^{2}} \xi\right\} \quad \text { if }\left|\varphi_{2}(x)\right| \neq 0 \text { and }\left|\Phi_{t}(x)\right| \neq 0
\end{aligned}
$$


In turn, choose $\xi^{\prime \prime}>0$ with the following properties:

(2.7) $\quad \xi^{\prime \prime}<\xi^{\prime}$

(2.10) $\quad \xi^{\prime \prime}<\min \left\{\frac{\left|\varphi_{2}(x)\right|^{2}}{4\left|\Phi_{t}(x)\right|} \xi^{\prime}, \frac{\left|\Phi_{t}(x)\right|^{2}}{4\left|\varphi_{2}(x)\right|} \xi^{\prime}\right\} \quad$ if $\left|\varphi_{2}(x)\right| \neq 0$ and $\left|\Phi_{t}(x)\right| \neq 0$.

Note that the functions $X \rightarrow \mathbb{R}_{\geq 0}: x \mapsto\left|\varphi_{2}(x)\right|$ and $X \times I \rightarrow \mathbb{R}_{\geq 0}:(x, t) \mapsto$ $\left|\Phi_{t}(x)\right|$ are continuous. So, there is $\delta_{2}>0$ such that if $\mathrm{d}\left(x, x^{\prime}\right)<\delta_{2}\left(x^{\prime} \in X\right)$ and $\left|t-t^{\prime}\right|<\delta_{2}\left(t^{\prime} \in I\right)$, then

$$
\begin{aligned}
& || \varphi_{2}(x)|-| \varphi_{2}\left(x^{\prime}\right)||<\xi^{\prime \prime}, \\
& || \Phi_{t}(x)|-| \Phi_{t^{\prime}}\left(x^{\prime}\right)||<\xi^{\prime \prime} .
\end{aligned}
$$

Using (2.7)-(2.12), we find that whenever $\mathrm{d}\left(x, x^{\prime}\right)<\delta_{2}\left(x^{\prime} \in X\right)$ and $\left|t-t^{\prime}\right|<$ $\delta_{2}\left(t^{\prime} \in I\right)$, then

$$
\begin{aligned}
& \left|\frac{1}{\mu(x, t)}-\frac{1}{\mu\left(x^{\prime}, t^{\prime}\right)}\right|<\xi^{\prime} \quad \text { if }\left|\varphi_{2}(x)\right| \neq 0, \\
& \left|\frac{1}{\nu(x, t)}-\frac{1}{\nu\left(x^{\prime}, t^{\prime}\right)}\right|<\xi^{\prime} \quad \text { if }\left|\Phi_{t}(x)\right| \neq 0 .
\end{aligned}
$$

Let $\delta=\min \left\{\delta_{1}, \delta_{2}\right\}$, and suppose that $\left(x^{\prime}, t^{\prime}\right) \in X \times I$ satisfies $\mathrm{d}\left(x, x^{\prime}\right)<\delta$ and $\left|t-t^{\prime}\right|<\delta$. We wish to show

$$
\mathrm{d}\left(\Psi_{2}(x, t)(u), \Psi_{2}\left(x^{\prime}, t^{\prime}\right)(u)\right)<\varepsilon \quad \text { for any } u \in I .
$$

CASE 1: $\left|\varphi_{2}(x)\right| \neq 0$ and $\left|\Phi_{t}(x)\right| \neq 0$. Without loss of generality, we can assume $\left|\varphi_{2}\left(x^{\prime}\right)\right| \neq 0$ and $\left|\Phi_{t^{\prime}}\left(x^{\prime}\right)\right| \neq 0$.

(1) If $0 \leq u \leq \mu\left(x^{\prime}, t^{\prime}\right)<\mu(x, t)$ or $0 \leq u \leq \mu(x, t)<\mu\left(x^{\prime}, t^{\prime}\right)$, then by (2.1), (2.3) and (2.13),

$$
\begin{aligned}
& \mathrm{d}\left(\varphi_{2}(x)\left(\frac{u}{\mu(x, t)}\right), \varphi_{2}\left(x^{\prime}\right)\left(\frac{u}{\mu\left(x^{\prime}, t^{\prime}\right)}\right)\right) \\
& \leq \mathrm{d}\left(\varphi_{2}(x)\left(\frac{u}{\mu(x, t)}\right), \varphi_{2}(x)\left(\frac{u}{\mu\left(x^{\prime}, t^{\prime}\right)}\right)\right) \\
& \quad+\mathrm{d}\left(\varphi_{2}(x)\left(\frac{u}{\mu\left(x^{\prime}, t^{\prime}\right)}\right), \varphi_{2}\left(x^{\prime}\right)\left(\frac{u}{\mu\left(x^{\prime}, t^{\prime}\right)}\right)\right)<\varepsilon .
\end{aligned}
$$


(2) If $\mu\left(x^{\prime}, t^{\prime}\right)<u \leq \mu(x, t)$, then by (2.1), (2.2), (2.4), (2.6), and (2.13),

$$
\begin{aligned}
& \mathrm{d}\left(\varphi_{2}(x)\left(\frac{u}{\mu(x, t)}\right), \Phi_{t^{\prime}}\left(x^{\prime}\right)\left(\frac{u-\mu\left(x^{\prime}, t^{\prime}\right)}{\nu\left(x^{\prime}, t^{\prime}\right)}\right)\right) \\
& \leq \mathrm{d}\left(\varphi_{2}(x)\left(\frac{u}{\mu(x, t)}\right), \varphi_{2}(x)(1)\right) \\
& \quad+\mathrm{d}\left(\Phi_{t}(x)(0), \Phi_{t}(x)\left(\frac{u-\mu\left(x^{\prime}, t^{\prime}\right)}{\nu\left(x^{\prime}, t^{\prime}\right)}\right)\right) \\
& \quad+\mathrm{d}\left(\Phi_{t}(x)\left(\frac{u-\mu\left(x^{\prime}, t^{\prime}\right)}{\nu\left(x^{\prime}, t^{\prime}\right)}\right), \Phi_{t^{\prime}}\left(x^{\prime}\right)\left(\frac{u-\mu\left(x^{\prime}, t^{\prime}\right)}{\nu\left(x^{\prime}, t^{\prime}\right)}\right)\right)<\varepsilon .
\end{aligned}
$$

(3) If $\mu(x, t)<u \leq \mu\left(x^{\prime}, t^{\prime}\right)$, then by (2.1)-(2.3), (2.6), and (2.13),

$$
\begin{aligned}
& \mathrm{d}\left(\Phi_{t}(x)\left(\frac{u-\mu(x, t)}{\nu(x, t)}\right), \varphi_{2}\left(x^{\prime}\right)\left(\frac{u}{\mu\left(x^{\prime}, t^{\prime}\right)}\right)\right) \\
& \leq \mathrm{d}\left(\Phi_{t}(x)\left(\frac{u-\mu(x, t)}{\nu(x, t)}\right), \Phi_{t}(x)(0)\right)+\mathrm{d}\left(\varphi_{2}(x)(1), \varphi_{2}(x)\left(\frac{u}{\mu\left(x^{\prime}, t^{\prime}\right)}\right)\right) \\
& \quad+\mathrm{d}\left(\varphi_{2}(x)\left(\frac{u}{\mu\left(x^{\prime}, t^{\prime}\right)}\right), \varphi_{2}\left(x^{\prime}\right)\left(\frac{u}{\mu\left(x^{\prime}, t^{\prime}\right)}\right)\right)<\varepsilon .
\end{aligned}
$$

(4) If $\mu\left(x^{\prime}, t^{\prime}\right)<\mu(x, t)<u \leq 1$ or $\mu(x, t)<\mu\left(x^{\prime}, t^{\prime}\right)<u \leq 1$, then by (2.2), (2.4), (2.6), (2.13), and (2.14),

$$
\begin{aligned}
\mathrm{d}\left(\Phi_{t}(x)\right. & \left.\left(\frac{u-\mu(x, t)}{\nu(x, t)}\right), \Phi_{t^{\prime}}\left(x^{\prime}\right)\left(\frac{u-\mu\left(x^{\prime}, t^{\prime}\right)}{\nu\left(x^{\prime}, t^{\prime}\right)}\right)\right) \\
\leq & \mathrm{d}\left(\Phi_{t}(x)\left(\frac{u-\mu(x, t)}{\nu(x, t)}\right), \Phi_{t}(x)\left(\frac{u-\mu\left(x^{\prime}, t^{\prime}\right)}{\nu\left(x^{\prime}, t^{\prime}\right)}\right)\right) \\
& +\mathrm{d}\left(\Phi_{t}(x)\left(\frac{u-\mu\left(x, t^{\prime}\right)}{\nu\left(x^{\prime}, t^{\prime}\right)}\right), \Phi_{t^{\prime}}\left(x^{\prime}\right)\left(\frac{u-\mu\left(x^{\prime}, t^{\prime}\right)}{\nu\left(x^{\prime}, t^{\prime}\right)}\right)\right)<\varepsilon .
\end{aligned}
$$

CASE 2: $\left|\varphi_{2}(x)\right|=0$ and $\left|\Phi_{t}(x)\right| \neq 0$.

(1) If $0 \leq u \leq \mu\left(x^{\prime}, t^{\prime}\right)$, then by (2.4), (2.9), (2.11), and (2.12),

$$
\begin{aligned}
& \mathrm{d}\left(\Phi_{t}(x)(u), \varphi_{2}\left(x^{\prime}\right)\left(\frac{u}{\mu\left(x^{\prime}, t^{\prime}\right)}\right)\right) \\
& \leq \mathrm{d}\left(\Phi_{t}(x)(u), \Phi_{t}(x)(0)\right)+\mathrm{d}\left(\Phi_{t}(x)(0), \Phi_{t^{\prime}}\left(x^{\prime}\right)(0)\right) \\
&+\mathrm{d}\left(\varphi_{2}\left(x^{\prime}\right)(1), \varphi_{2}\left(x^{\prime}\right)\left(\frac{u}{\mu\left(x^{\prime}, t^{\prime}\right)}\right)\right)<\varepsilon
\end{aligned}
$$


(2) If $\mu\left(x^{\prime}, t^{\prime}\right)<u \leq 1$, then by (2.2), (2.4), (2.9), (2.11), and (2.12),

$$
\begin{aligned}
& \mathrm{d}\left(\Phi_{t}(x)(u), \Phi_{t^{\prime}}\left(x^{\prime}\right)\left(\frac{u-\mu\left(x^{\prime}, t^{\prime}\right)}{\nu\left(x^{\prime}, t^{\prime}\right)}\right)\right) \\
& \leq \mathrm{d}\left(\Phi_{t}(x)(u), \Phi_{t}(x)\left(\frac{u-\mu\left(x^{\prime}, t^{\prime}\right)}{\nu\left(x^{\prime}, t^{\prime}\right)}\right)\right) \\
& \quad+\mathrm{d}\left(\Phi_{t}(x)\left(\frac{u-\mu\left(x^{\prime}, t^{\prime}\right)}{\nu\left(x^{\prime}, t^{\prime}\right)}\right), \Phi_{t^{\prime}}\left(x^{\prime}\right)\left(\frac{u-\mu\left(x^{\prime}, t^{\prime}\right)}{\nu\left(x^{\prime}, t^{\prime}\right)}\right)\right)<\varepsilon .
\end{aligned}
$$

CASE $3:\left|\varphi_{2}(x)\right| \neq 0$ and $\left|\Phi_{t}(x)\right|=0$.

(1) If $0 \leq u \leq \mu\left(x^{\prime}, t^{\prime}\right)$, then by (2.1), (2.3) and (2.13),

$$
\begin{aligned}
\mathrm{d}\left(\varphi_{2}(x)(u), \varphi_{2}\left(x^{\prime}\right)(\right. & \left.\left.\frac{u}{\mu\left(x^{\prime}, t^{\prime}\right)}\right)\right) \leq \mathrm{d}\left(\varphi_{2}(x)(u), \varphi_{2}(x)\left(\frac{u}{\mu\left(x^{\prime}, t^{\prime}\right)}\right)\right) \\
& +\mathrm{d}\left(\varphi_{2}(x)\left(\frac{u}{\mu\left(x^{\prime}, t^{\prime}\right)}\right), \varphi_{2}\left(x^{\prime}\right)\left(\frac{u}{\mu\left(x^{\prime}, t^{\prime}\right)}\right)\right)<\varepsilon .
\end{aligned}
$$

(2) If $\mu\left(x^{\prime}, t^{\prime}\right)<u \leq 1$, then by (2.1), (2.3) (2.8), (2.11), and (2.12),

$$
\begin{aligned}
\mathrm{d}\left(\varphi_{2}(x)(u), \Phi_{t^{\prime}}\left(x^{\prime}\right)\left(\frac{u-\mu\left(x^{\prime}, t^{\prime}\right)}{\nu\left(x^{\prime}, t^{\prime}\right)}\right)\right) \\
\leq \mathrm{d}\left(\varphi_{2}(x)(u), \varphi_{2}(x)(1)\right)+\mathrm{d}\left(\varphi_{2}(x)(1), \varphi_{2}\left(x^{\prime}\right)(1)\right) \\
\quad+\mathrm{d}\left(\Phi_{t^{\prime}}\left(x^{\prime}\right)(0), \Phi_{t^{\prime}}\left(x^{\prime}\right)\left(\frac{u-\mu\left(x^{\prime}, t^{\prime}\right)}{\nu\left(x^{\prime}, t^{\prime}\right)}\right)\right)<\varepsilon .
\end{aligned}
$$

CASE 4: $\left|\varphi_{2}(x)\right|=0$ and $\left|\Phi_{t}(x)\right|=0$.

(1) If $0 \leq u \leq \mu\left(x^{\prime}, t^{\prime}\right)$, then by (2.3),

$$
\begin{aligned}
& \mathrm{d}\left(\varphi_{2}(x)(0), \varphi_{2}\left(x^{\prime}\right)\left(\frac{u}{\mu\left(x^{\prime}, t^{\prime}\right)}\right)\right) \\
& \quad \leq \mathrm{d}\left(\varphi(x)\left(\frac{u}{\mu\left(x^{\prime}, t^{\prime}\right)}\right), \varphi\left(x^{\prime}\right)\left(\frac{u}{\mu\left(x^{\prime}, t^{\prime}\right)}\right)\right)<\varepsilon .
\end{aligned}
$$

(2) If $\mu\left(x^{\prime}, t^{\prime}\right)<u \leq 1$, then by (2.4) and (2.12),

$$
\begin{aligned}
\mathrm{d}\left(\varphi_{2}(x)(0), \Phi_{t^{\prime}}\left(x^{\prime}\right)(\right. & \left.\left.\frac{u-\mu\left(x^{\prime}, t^{\prime}\right)}{\nu\left(x^{\prime}, t^{\prime}\right)}\right)\right) \leq \mathrm{d}\left(\Phi_{t}(x)(1), \Phi_{t^{\prime}}\left(x^{\prime}\right)(1)\right) \\
& +\mathrm{d}\left(\Phi_{t^{\prime}}\left(x^{\prime}\right)(1), \Phi_{t^{\prime}}\left(x^{\prime}\right)\left(\frac{u-\mu\left(x^{\prime}, t^{\prime}\right)}{\nu\left(x^{\prime}, t^{\prime}\right)}\right)\right)<\varepsilon .
\end{aligned}
$$

Theorem 2.2. For any pull-back diagram

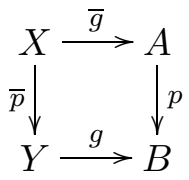


if $p$ has the SHLP with respect to every space, and if $A, B$, and $Y$ are $A N R$ 's, then $X$ is an ANR.

Proof. Suppose that $f: C \rightarrow X$ is a map from a closed subset $C$ of a space $Z$ into $X$. We wish to show that $f$ has an extension over some open neighborhood $U$ of $C$.

Let $\mathcal{V}$ be an open covering of $B$ so that any two $\mathcal{V}$-near maps $\varphi, \psi: W \rightarrow$ $B$ admit a homotopy $H: W \times I \rightarrow B$ which connects them and is constant on $x \times I$ whenever $\varphi(x)=\psi(x)$, and let $\mathcal{V}^{\prime}$ be an open covering of $B$ such that st $\mathcal{V}^{\prime}<\mathcal{V}$. Since $A$ and $Y$ are ANR's, the maps $\bar{g} f: C \rightarrow A$ and $\bar{p} f: C \rightarrow Y$ extend to maps $f_{1}^{\prime}: U^{\prime} \rightarrow A$ and $f_{2}^{\prime}: U^{\prime} \rightarrow Y$ for some open neighborhood $U^{\prime}$ of $C$. Let $\mathcal{U}=f_{1}^{\prime-1} p^{-1} \mathcal{V}^{\prime} \wedge f_{2}^{\prime-1} g^{-1} \mathcal{V}^{\prime}$, and let $U=\operatorname{st}(C, \mathcal{U})$. If $f_{1}: U \rightarrow A$ and $f_{2}: U \rightarrow Y$ are the restrictions of $f_{1}^{\prime}$ and $f_{2}^{\prime}$, respectively, then $\left(p f_{1}, g f_{2}\right)<\mathcal{V}$. Indeed, for each $x \in U$, there exist $c \in C$ and $V_{1}, V_{2} \in \mathcal{V}^{\prime}$ such that both $x$ and $c$ belong to $f_{1}^{-1} p^{-1}\left(V_{1}\right) \wedge f_{2}^{-1} g^{-1}\left(V_{2}\right)$. Then $p f_{1}(c)=g f_{2}(c) \in V_{1} \cap V_{2} \neq \emptyset, p f_{1}(x) \in V_{1}$, and $g f_{2}(x) \in V_{2}$, which implies that $p f_{1}(x), g f_{2}(x) \in V_{1} \cup V_{2} \subseteq V$ for some $V \in \mathcal{V}$ as required. By the choice of $\mathcal{V}, p f_{1} \simeq g f_{2}$. Since $p$ has the SHLP, there is a map $f_{1}^{\prime \prime}: U \rightarrow A$ such that $f_{1}^{\prime \prime} \simeq f_{1}, p f_{1}^{\prime \prime}=g f_{2}$, and $f_{1}^{\prime \prime}(x)=f_{1}(x)$ whenever $p f_{1}(x)=g f_{2}(x)$ for $x \in U$. The maps $f_{1}^{\prime \prime}$ and $f_{2}$ define a map $\tilde{f}: U \rightarrow X$ such that $\bar{g} \tilde{f}=f_{1}^{\prime \prime}$ and $\bar{p} \tilde{f}=f_{2}$. Moreover, if $x \in C$, then $p f_{1}(x)=g f_{2}(x)$, so $f_{1}^{\prime \prime}(x)=f_{1}(x)$, and hence $\tilde{f}$ is an extension of $f$. This proves that $X$ is an ANR.

THEOREM 2.3. For each 2 -sink $Y \stackrel{g}{\rightarrow} B \stackrel{p}{\leftarrow} A$ in ANR with $f$ having the SHLP with respect to every space, there is a pull-back diagram

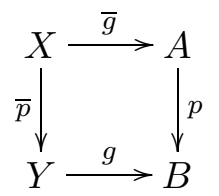

in ANR with $\bar{p}$ having the SHLP with respect to every space. Moreover, if $p$ (resp., $g$ ) is a homotopy equivalence, so is $\bar{p}$ (resp., $\bar{g}$ ).

Proof. The existence of the pull-back diagram follows from Theorem 2.2. That $\bar{p}$ has the SHLP with respect to every space easily follows. The second assertion follows from the case of Top.

THEOREM A. ANR is a fibration category if fibration = map having the SHLP with respect to every space, and weak equivalence = homotopy equivalence.

Proof. (F2) and (F3) follow from Theorems 2.3 and 2.2, respectively. (F1) and (F4) follow from those properties for Top. 


\section{Proof of Theorem B}

THEOREM 3.1. Every map $f: A \rightarrow B$ between metric spaces is the composition of a homotopy equivalence $q: A \rightarrow E$ and a map $p: E \rightarrow B$ having the SHLP with respect to every space, where $E$ is some metric space.

Proof. Let $E=\left\{(x, \omega) \in A \times B^{I}: f(x)=\omega(0)\right\}$. Then $E$ is a metric space. The map $f$ factors into the composition of $q: A \rightarrow E$ and $p: E \rightarrow B$ which are defined as in the proof of Theorem 2.1. Then $q$ is a homotopy equivalence. Indeed, if $p_{A}: E \rightarrow A$ is the restriction of the projection map of $A \times B^{I}$ onto $A$, then $p_{A} p=1_{A}$, and $p p_{A} \simeq 1_{E}$ with the homotopy $H: E \times I \rightarrow E, H((x, \omega), t)=\left(x, \omega_{t}\right)$. That $p$ has the SHLP with respect to any space $X$ is proven similarly to Theorem 2.1.

TheOREM B. The full subcategory $\mathrm{M}$ of Top whose objects are metric spaces is a fibration category if fibration = map having the SHLP with respect to every space, and weak equivalence = homotopy equivalence.

Proof. (F2) easily follows, and (F3) follows from Theorem 3.1. (F1) and (F4) follow from those properties for Top.

REMARK. We observe that the Eckmann-Hilton duality breaks down when taking subcategories. Top has the structures of a fibration category and a cofibration category, but the restriction of the cofibration structure to $\mathrm{M}$ is not a cofibration category, while the restriction of the fibration structure to $\mathrm{M}$ is a fibration category.

\section{References}

[1] H. J. Baues, Algebraic Homotopy, Cambridge Univ. Press, Cambridge, 1989.

[2] S. Mardešić and J. Segal, Shape Theory, North-Holland, 1982.

[3] D. G. Quillen, Homotopical Algebra, Lecture Notes in Math. 43, Springer, 1967.

[4] E. H. Spanier, Algebraic Topology, McGraw-Hill, New York, 1966.

Takahisa Miyata

Department of Mathematics and Informatics

Graduate School of Human Development and Environment

Kobe University

Kobe, 657-8501, Japan

E-mail: tmiyata@kobe-u.ac.jp 